In this small series of 20 relapses ( 11 detected by non-routine marrow examination, 9 by routine examination), 8 children in the first group and 6 in the second group achieved a second remission.

Because of the small number of patients no useful comment can be made about the duration of the second remission. However, in view of the overall poor prognosis of children with ALL who have bone marrow relapses during treatment, ${ }^{4}$ or after treatment has stopped, ${ }^{5}$ it seems improbable that overall survival after relapse would be much, if at all, reduced by the abolition of routine marrow examinations. It should be stressed that each child in this study, by the design of the study, had had a normal marrow examination at least 12 weeks before.

The figures presented in this study show that routine marrow examination every 3 months in children with ALL or NHL has a low (0.4\%) relapse yield if the peripheral count is normal; even if the count is abnormal, without circulating blast cells, the yield only increases to a modest $12 \%$.

It is therefore suggested that routine marrow examination during maintenance therapy is not worth while and should not be included in the protocols of future studies. The clinician must obviously establish remission in a new case after induction therapy and should not hesitate to proceed to marrow examination in a child in remission whose peripheral counts indicate cause for concern.

It is hoped that the well-known trauma to child, parents, and doctor of performing a marrow examination in a conscious or poorly-sedated child will be lessened by the abolition of routine marrow examination. In addition the small, but ever present, risk of a general anaesthetic in those units fortunate enough to have such a service, will be lessened even further by the administration of fewer anaesthetics.

We thank Mrs M Coggin for secretarial assistance, and Staff Nurse $\mathbf{J}$ Young for help with the data extraction.

\section{References}

1 Hardisty R M, Chessells J M. Acute leukaemia in childhood. Management and prognosis. In: Hoffbrand A V, Brain M C, Hirsh J, eds. Recent advances in haematology. Vol. 2. Edinburgh: Churchill Livingstone, 1977: 159-73.

2 Medical Research Council Working Party on Leukaemia in Childhood. Effects of varying radiation schedule, cyclophosphamide treatment, and duration of treatment in acute lymphoblastic leukaemia. $\mathrm{Br} \mathrm{Med} J$ 1978; ii: 787-91.

3 Jones B, Klingberg W G. Lymphosarcoma in children. J Pediatr 1963; 63: 11-20.

4 Cornblect M A, Chessells J M. Bone marrow relapse in acute lymphoblastic leukaemia in childhood. $\mathrm{Br}$ Med $\mathrm{J}$ 1978; ii: 104-6.

5 Rivera G, Pratt C B, Aur J A R, Verzosa M, Hustu H O. Recurrent childhood lymphocytic leukaemia following cessation of therapy. Cancer 1976; 37: 1679-86.

Correspondence to Dr C C Bailey, Regional Paediatric Oncology Unit, Seacroft Hospital, York Road, Leeds LS14 6UH.

Reccived 8 May 1980

\title{
Congenital villous atrophy associated with stagnant loop syndrome
}

\section{J F B DOSSETOR}

Department of Child Health, Royal Hospital for Sick Children, Glasgow

SUMMARY A child who presented at age 9 months with steatorrhoea and malnutrition is described. After an initial period of intravenous feeding it was found that oral gentamicin led to a reduction of clinical steatorrhoea and an increase in weight, and so gentamicin was continued for 18 months. Investigation showed severe villous atrophy without pronounced inflammatory cell infiltrate and with no increase in intraepithelial lymphocytes. The villous atrophy was not present in the duodenum but started in the jejunum. The small-intestine was radiologically dilated throughout its length. It is suggested that this structurally-abnormal gut acted as a stagnant loop and exacerbated the steatorrhoea.
Intractable diarrhoea of infancy has many causes ${ }^{1}$ and a high mortality. The child described in this report had intractable diarrhoea and presented with malnutrition at age 9 months. Her life was saved by a period of intravenous feeding. Subsequent investigation showed an unusual enteropathy.

\section{Case report}

This baby girl was born at 32 weeks' gestational age (birthweight $1.56 \mathrm{~kg}$ ) by spontaneous vertex delivery and there were no neonatal problems. At age 2 months, she was discharged home with a weight of $2.41 \mathrm{~kg}$. There had been no diarrhoea and she was fed on Cow and Gate Baby Milk Plus. 
About one month after discharge from hospital her stools became abnormal; they were loose, pale, and foul-smelling. No mixed feeds or cereals had been given her. There was no vomiting and her appetite was good but during the next 6 months she became emaciated with a protuberant abdomen; she was first admitted to hospital at age 9 months with a weight of $4 \cdot 58 \mathrm{~kg}$.

She remained in hospital for the next 4 months and at the time of first discharge her weight was $4 \cdot 18 \mathrm{~kg}$. A duodenal biopsy was performed which showed minor changes only. She had no gluten throughout the 4-month period and various dietary restrictions were tried including a disaccharide-free, and whole protein-free, diet based on Albumaid. None of these dietary manipulations led to a consistent weight gain.

Three months after discharge, at 15 months, she was readmitted as an emergency. She was emaciated with a weight of $4.44 \mathrm{~kg}$. She had had severe diarrhoea and vomiting for 3 days before admission which had led to dehydration. After resuscitation she was given a period of intravenous feeding (total 4 weeks). By the end of this period her weight had increased to $5 \cdot 28 \mathrm{~kg}$.

During the next month a series of investigations was performed. Stool microscopical examination and culture were repeatedly negative; sweat test (repeated 4 times) was normal; faecal fat excretion was high (42 mmol/24h; $11.9 \mathrm{~g} / 24 \mathrm{~h})$; xylose absorption 1 hour after $5 \mathrm{~g}$ oral dose was normal, $2.7 \mathrm{mmol} / 1(40.6 \mathrm{mg} / 100 \mathrm{ml})$; serum lipoprotein, faecal bile acid output, and plasma zinc and copper concentrations were normal. There was complete achlorhydria.

A duodenal biopsy showed minor change (grade 1) only, with no increase in intraepithelial lymphocytes; disaccharidase levels were normal; cryostat sections stained with oil-red-O showed no significant content of neutral lipid. Duodenal aspirate grew a heavy growth of Escherichia coli $\left(10^{5} / \mathrm{ml}\right)$, and Candida albicans $\left(10^{5} / \mathrm{ml}\right)$; the $\mathrm{pH}$ of the aspirate was 8.07 , bicarbonate $22 \mathrm{mmol} / \mathrm{l}$, amylase $21600 \mathrm{U} / \mathrm{l}$, lipase $292 \mathrm{U} / \mathrm{ml}$, trypsin present to a dilution of 1 in 800 . A comprehensive analysis of immune function was performed. Plasma immunoglobulin levels, serum complement, and opsonising capacity were all normal. T- and B-cell numbers were normal in peripheral blood, and lymphocyte transformation was in the low-normal range. IgA was present in duodenal aspirate in roughly normal amounts. Delayed cutaneous hypersensitivity reactions to common antigens were all negative. Barium followthrough showed a dilated small-bowel and very slow passage of barium. Antibodies to gluten were present in blood. Her HLA status was A2A3, B7B12, Bw4Bw6, Cw5.

Because of the jejunal aspirate cultures she was given a course of oral nystatin and then oral gentamicin. Immediately oral gentamicin was introduced she began to gain weight and showed catch-up growth until she reached the 3 rd centile for her age at 30 months.

During these months it became clear that her weight gain depended on the continuance of the oral antibiotic, either gentamicin or colistin. Fig. 1 summarises the faecal fat results. Faecal fat excretion was abnormal on every occasion except one, regardless of treatment. Twice there was a return of severe clinical steatorrhoea which responded within hours to the oral antibiotic being restarted. On the second of these occasions only, a 5-day stool collection was obtained immediately before and after restarting gentamicin, documenting an improvement in faecal fat excretion at the same time as symptomatic improvement. Stools had never been normal even when she was well, being pale and semiformed, and passed once or twice a day.

At 29 months 3 intestinal biopsies were taken from the third part of duodenum, duodenojejunal junction, and jejunum. The histology of the abnormal specimens is shown in Fig. 2. The duodenal biopsy showed minor change only; the junctional biopsy showed severe partial villous atrophy; the most distal biopsy showed subtotal villous atrophy, however there was only minor inflammatory infiltrate and no increase in intraepithelial lymphocytes. Electron microscopical examination of a fragment of the proximal biopsy showed normal enterocytes with a normal brush border.

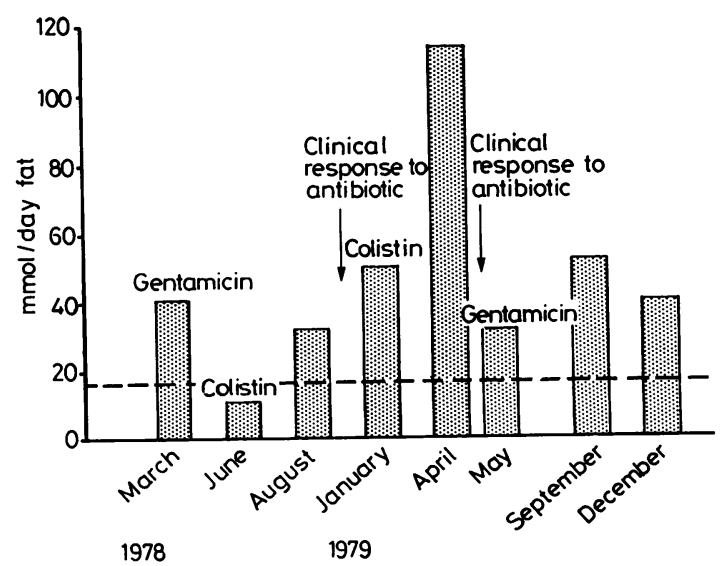

Fig. 1 Faecal fat output in relation to treatment with oral antibiotics. Dotted line shows upper limit of normal daily stool fat content. 


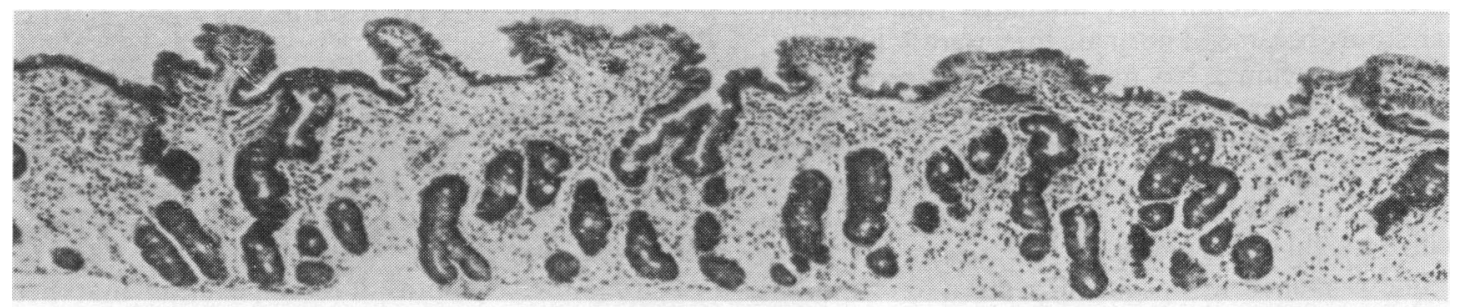

(a)

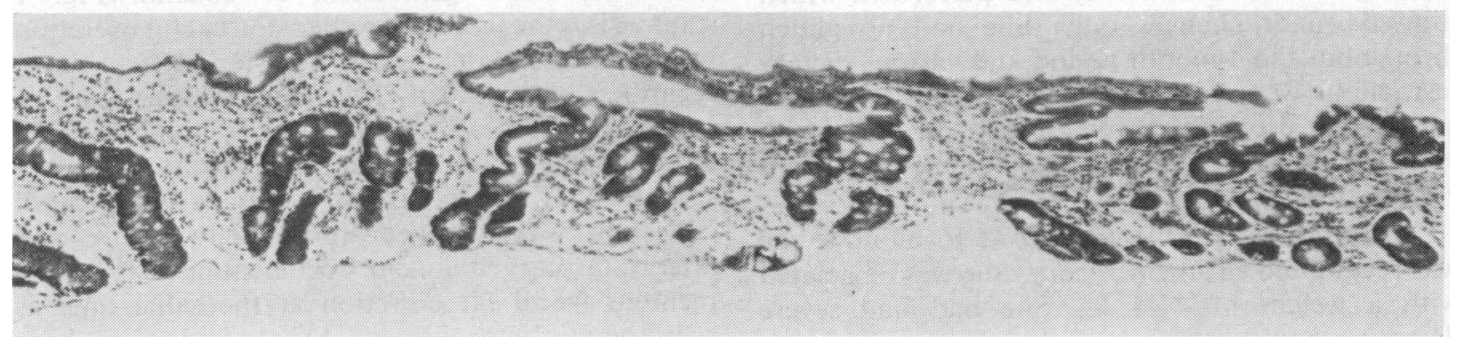

(b)

Fig. 2 Histology of intestinal biopsies from (a) duodeno-jejunal junction showing severe partial villous atrophy, and (b) jejunum showing subtotal villous atrophy.

Subsequently, investigations have shown a return of normal gastric acid output, and an improvement in the radiological appearance of the small-bowel with a normal rate of passage of barium. At present she is neurodevelopmentally normal despite the severe malnutrition earlier, and she is on the 3rd centile for height and weight. She still has a slightly distended abdomen. A recent jejunal biopsy (at $4 \frac{1}{2}$ years) has shown improvement in her villous morphology but the villi remain small. The only additional problem has been the development of a left staghorn renal calculus, associated with urinary tract infection, both of which have been treated. The calculus formation has probably resulted from excessive colonic oxalate absorption, a non-specific effect of chronic steatorrhoea. ${ }^{2}$

\section{Discussion}

At the time of the initial investigations the child was extremely malnourished. Some of the results obtained then probably related to the nutritional state rather than to primary manifestations of disease, in particular the achlorhydria ${ }^{3}$ (which has subsequently recovered), and the fairly minor changes in immune function. ${ }^{4}$

Two duodenal biopsies were performed, at ages 10 and 16 months. Each showed minor change. It was only when a series of upper small intestinal biopsies was obtained at age 29 months, and mucosa distal to the duodenum was sampled, that a severe mucosal enteropathy was diagnosed. This started at the level of the proximal jejunum. In intestinal hypersensitivity states the changes are as diagnostic in duodenum as in jejunum, and hence it was not appreciated initially that the first 2 biopsies might not be representative.

The mucosal changes are unlikely to have been caused by intraluminal events. Symptoms started at age 3 months when the only dietary constituent was milk. At the time of her first admission there was no clinical improvement on cows' milk protein-free diet. It is also certain that the child is not hypersensitive to gluten as, apart from atypical histological features, symptoms began before gluten was introduced into her diet, and during both times she was in hospital there was no response to the withdrawal of gluten from her diet. Although small intestinal bacteria can cause mucosal change it is generally a partial rather than subtotal villous atrophy. ${ }^{5}$ Moreover, if the villous atrophy were induced by bacteria or their toxic metabolites, it should affect the duodenum as much as the jejunum.

It is suggested that this child has a congenital villous atrophy but that there was not symptomatic steatorrhoea in infancy until small intestinal bacterial overgrowth further compromised absorption at age 3 months. It is likely that the structural abnormality was accompanied by 
abnormal motility, at least in infancy, and the very slow passage of barium in the barium follow-through at age 18 months supports this. The abnormality in motility would be the main factor that predisposed to small intestinal bacterial overgrowth, but the secondary acquisition of achlorhydria would exacerbate this. At the time of her acute admission at age 15 months a secondary acute enterocolitis was present too. We have not been able to demonstrate a primary immune defect. It seems likely that some malabsorption will be a permanent feature of this child's life, but at the moment her increased appetite enables her to absorb enough to thrive despite persistent steatorrhoea.

I thank Dr W R McAinsh and Professor F Cockburn for permission to report this case.

\section{References}

1 Larcher V F, Shepherd R, Francis D E M, Harries J T. Protracted diarrhoea in infancy. Arch Dis Child 1977; 52: 597-605.

2 Ogilvie D, McCollum J P K, Packer S, et al. Urinary outputs of oxalate, calcium, and magnesium in children with $9 . \sqrt{ }$ intestinal disorders. Potential cause of renal calculi. Arch Dis Child 1976; 51 : 790-5.

3 Gracey M, Cullity G J, Suharjono, Sunoto. The stomach in malnutrition. Arch Dis Child 1977; 52: 325-7.

4 Smythe P M, Schonland M, Brereton-Stiles G G, et al. Thymolymphatic deficiency and depression of cell mediated immunity in protein-calorie malnutrition. Lancet 1971; ii: 939-44.

5 Kenworthy R. Effect of Escherichia coli on germ-free and gnotobiotic pigs. I. Light and electron microscopy of the small intestine. J Comp Pathol 1970; 80: 53-63.

Correspondence to Dr J F B Dossetor, Queen Elizabeth Hospital, Gayton Road, King's Lynn, Norfolk PE30 4ET.

Received 19 June 1980

\title{
Delayed separation of the umbilical cord, severe widespread infections, and immunodeficiency
}

\author{
J G BISSENDEN, M R HAENEY, M J TARLOW, AND R A THOMPSON
}

\begin{abstract}
Department of Paediatrics and Child Health, and Regional Immunology Laboratory, East Birmingham Hospital, Birmingham
\end{abstract}

SUMMARY Three of 4 children in a family died from infection before age one year. All 3 had delayed separation of the umbilical cord in the third week. One child was found to have defects both in specific antibody production and in neutrophil function; these were not improved by treatment with ascorbic acid or levamisole.

Hayward et al. ${ }^{1}$ described a new familial syndrome of delayed separation of the umbilical cord, widespread infections, and defective neutrophil mobility. They suggested that the isolated defect in neutrophil mobility could be corrected in vitro and in vivo by ascorbic acid. We report a family with an apparently similar clinical syndrome; however, in the child studied the immunological defect was more extensive and affected both neutrophil function and specific antibody production, and was not improved by treatment with ascorbic acid or levamisole.

\section{Case report}

A girl weighing $3 \mathrm{~kg}$ was born by caesarean section. She was the fourth child of healthy unrelated white parents. After slight neonatal asphyxia, she fed poorly from birth, and developed diarrhoea during the first week of life. The umbilical cord did not separate until the third week, leaving an imperfectly healed stump. At age 4 weeks she developed an axillary abscess which healed spontaneously, followed by a staphylococcal abscess on the chest wall requiring surgical incision and a course of flucloxacillin. She had a mild anaemia with a total white cell count of $45 \pm 10^{9} / 1$, of which $90 \%$ were neutrophils.

The family history is remarkable. The first child had died suddenly after 10 weeks, having previously been well; necropsy examination showed clostridial septicaemia due to gangrenous ileo-caecitis. The second child had died at age 7 weeks from overwhelming staphylococcal infection and necropsy had shown peritonitis complicating necrotising enterocolitis. The umbilical cords of both children had failed to separate until the third week of life. Both parents and the third sibling, whose cord separated normally, are well.

In view of this family history of unusual infection, the patient was transferred for immunological investigation at age 2 months, as a possible case of 